\title{
An Intelligent Parking Scheduling Algorithm based on Traffic and Driver Behavior Predictions
}

\author{
jiazao Lin ', Shi-Yong Chen', Chih-Yung Chang "2, Guilin Chen" \\ 'Peking University, Beijing 100871, China \\ ${ }^{2}$ Tamkang University, New Taipei City 25137, Taiwan \\ Chuzhou University, Chuzhou 239000, China \\ Linjz84@126.com \\ dora@gms.tku.edu.tw \\ cychang@mail.tku.edu.tw \\ glchen@chzu.edu.cn
}

\begin{abstract}
Smart parking is a common demand of citizen, especially for people living in a smart city. It is an important issue since it not only determines the required parking time of drivers but also impacts the urban population and traffic congestion. In this paper, an intelligent parking algorithm is presented based on the predictions of traffics and drivers' behaviors. The proposed parking algorithm analyzes the historical parking records, predicts the parking traffics and the driver's parking length and then schedules the vehicles to the parking grids such that the maximal benefits can be obtained. The proposed algorithm also dynamically allocates their reservations but guarantees the parking reservations for the VIP members. But based on the parking space resource. Performance analysis through extensive simulations demonstrates the efficiency and practicality of the proposed scheme.
\end{abstract}

\section{Introduction}

Parking lots are located all over the world. These parking lots were built in workplaces, sports centers, entertainment and tourist centers, shopping centers, airports, schools, family apartments and so on. The increments of cars are leading to more and larger parking lots, which increases efforts to deploy intelligent parking management systems. This also increases the motivation for developing an intelligent parking system for better managing parking space resources, providing a higher quality of parking service as well as increasing the benefits of the manager of the parking lots.

In the past few years, a number of researchers devoted themselves to improve the efficiency of parking systems. These studies developed new policies and algorithms, aiming for improving the utilization of parking grids or reduce the driver's parking time, creating advantages of the manager or drivers, respectively. These studies are generally partitioned into two classes. The first one [1-5] is free parking, which aims to provide parking information for drivers to easily park their vehicles to the parking grids of the street. The policy considered in this class is that the parking vehicle is free on the street. On the contrary, the second class is paid parking, which asked drivers to pay money for parking vehicle. Study [6] proposed an algorithm to allocate the incoming vehicles to available parking grids. It aims to improve the utilization of parking space 
such that the benefits of a manager can be maximized. However, most of them did not consider the predictions of parking traffics and drivers' behaviors based on parking history.

This paper proposed a new intelligent parking algorithm which considers the general needs of VIP members, drivers' service quality and the manager's benefits. Different from the previous works, the proposed algorithm predicts the traffics and the length of each driver's parking duration based on parking history. According to the predictions, the proposed parking algorithm selects the oncoming parking vehicles, schedules the parking grids and then allocates vehicles to the parking grids, aiming at maximizing the grid utilization and manager's benefits. The proposed algorithm not only guarantees the parking reservations for the VIP members but also improves the utilization of parking space.

To achieve this, the proposed algorithms consider two issues when developing the intelligent parking algorithm. The first one is the space reserved for VIP members. The VIP members have been usually maintained in the most parking management system. These members prepaid the money and expected to always have reservations of parking grids for their needs. To meet this requirement, the proposed algorithm considers the VIP members and dynamically reserves parking grids for them. The presented algorithm analyzes the behaviors of VIP members, predicts the starting parking time and length of time duration and then dynamically reserves the parking grids for them. In addition to the issue of VIP member, another important issue is the utilization of parking space. The proposed scheme aims to maximize the benefits for the manager of the parking lots.

The remainder of this paper is organized as follows. The assumptions, constraints and the design of the parking algorithm are proposed in the second section. The performance studies of the proposed algorithm and its improvements against the existing works are investigated in Section 3. Finally, the conclusions are drawn.

\section{The Proposed Parking Algorithm}

\subsection{Assumptions, Goal and Constraints}

This paper assumes that the information of drivers, their vehicles and their parking records have been collected in the parking management system. Each parking record contains the vehicle ID, starting parking time and end parking time. The parking time length of each parking record can be calculated based on this information. Given a parking lot which has k parking grids. A constant benefit can be created if the grid has been parked by vehicle for one basic time unit. Given a time period represented as $T=\left[t_{1}, t_{2}\right]$ where $t_{1}$ is a past time point and $t_{2}$ is a future time point. The overall benefits created by the parking lot is the summation of all benefits created by each parking grid in al he parking lot from $t_{1}$ to $t_{2}$. The goal of this paper aims to develop a parking schedule algorithm such that the overall benefits can be maximized. There are some constraints needed to be satisfied when finding the solution of the investigated issue. First, each parking grid can only have two states, the available state or the occupied state. Another constraint is that any vehicle can only occupy at most one parking grid at any given time in T. Similarly, each grid can only be occupied by at most one vehicle at any given time.

\subsection{The proposed algorithm}

The proposed algorithm is organized as three major parts. The first part mainly checks the validation of the input data and deletes the invalid ones existed in the historical parking records. After that, the designed algorithm further analyzes the history of parking records. This helps predict the parking traffic for a given specific day and time. Another important task in the second part is the behavior analysis, which aims to estimate the length of parking time for a certain driver. This analysis can help better schedule the parking grid and predict the time of an occupied grid to be released. Based on these predictions, the third part further selects proper vehicles from the incoming vehicles and then allocates them to the parking grids which are available. 
There are three different policies proposed in the parking algorithm, namely Basic-Best-Fit, Basic-Worst-Fit and Parking Behavior Forecast. The designing concept of the Basic-Worst-Fit is to identify the starting time of each parking grid and guide the incoming vehicle to the grid with the largest available duration. The second policy, called Basic-Worst-Fit, guides the incoming vehicle to the parking grid whose available duration best matches the predicted length of the vehicle. The last part, called Parking Behavior Forecast (PBF) Scheme, predicts parking behavior based on parking history and the traffic for any given time of each weekday. The implementation of the Parking Behavior Forecast algorithm is separated into three parts. The first one is to fill in the VIP members to the empty parking grids. Then the method selects proper vehicles when the number of incoming vehicles larger than the number of available parking grids. The third one is to allocate the selected vehicles to the best-matched grids.

\section{Simulation and Results}

This section presents the performance evaluation of the proposed SPA method in terms of parking rates and the number of rejected vehicles. The proposed SPA is compared with traditional parking(TP) method [6]. The traditional parking method mainly adopted the strategy of FIFS (First comes First Serve). The proposed SPA adopts three policies, which are noted by BB-SPA, BW-SPA and PBF-SPA, respectively. The designing policies of the Basic-Best-Fit and the BasicWorst-Fit have been presented in the previous section.

As shown in Fig. 1, 17 to 18 o'clock has peak traffics from Monday to Friday. Fig. 2 shows that the weekends have peak traffic from 9 to 11 o'clock. However, the parking traffic of any given time points does not exceed 300 .

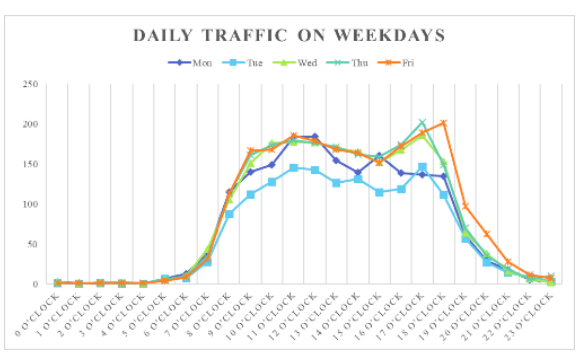

Fig. 1 Daily traffic from Monday to Friday

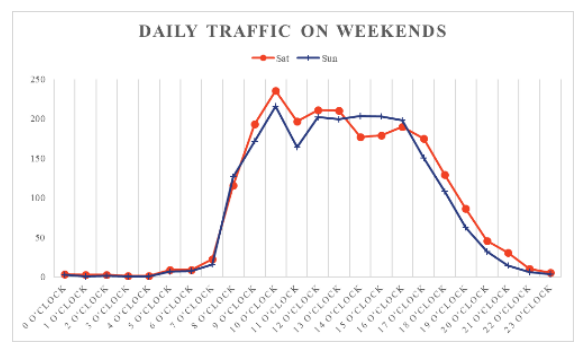

Fig. 2 Daily traffic from Saturday to Sunday. 
Figs. 3 and 4 studies the accumulated parking rate of the parking lot with 300 grids. The parking traffic is created by scaling the real parking traffic, ranging from $75 \%$ to $95 \%$. That is, the traffic is reduced ranging from $5 \%$ to $25 \%$. The proposed SPA and the existing TP have a similar trend that the accumulated parking rate of them increase with time but decrease with the proportion of traffic reduction. This outcome is because of low parking traffic, which causes low parking rate. The proposed PBF-SPA has the best performance, as compared with the other three algorithms in all cases, because that PBF-SPA further predicts the vehicle arrival time and the parking length, and allows VIPs booking parking grids. The BB-SPA has a better performance than BW-SPA on both weekdays and weekends. This is because that BB-SPA predicts the duration of parking time and it always guides the vehicle to the grid with appreciate available time length. Consequently, the grid which is available for a long time can be reserved for the vehicle with the need for parking for a long time. The BW-SPA always guides the vehicle to the grid with maximal available duration and partitions the available duration into several small time segments. When a vehicle needs to be parked for a long time, the BW-SPA cannot support this requirement.

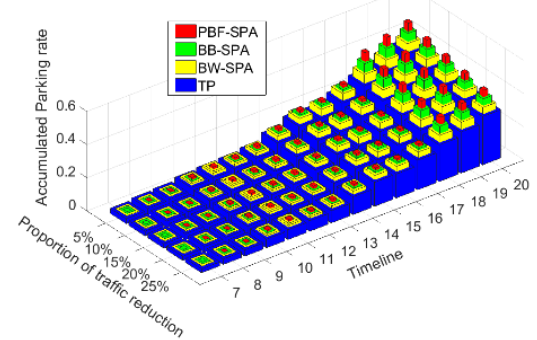

Fig. 3 Parking rate for different proportion of traffic reduction on weekdays.

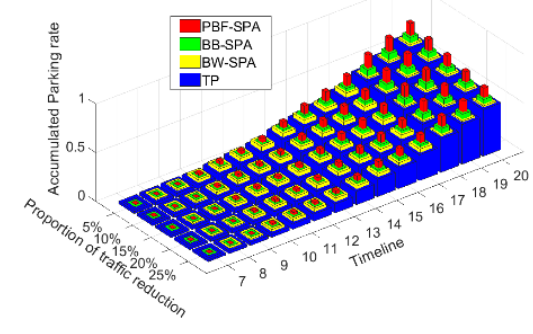

Fig. 4 Parking rate for different proportion of traffic reduction on weekdays.

\section{Conclusion}

This work proposes an intelligent parking algorithm with three scheduling policies, aiming to improve the parking rate and obtain the maximal profits. The proposed BW-SPA and BB-SPA adopt the worst fit and best-fit policies, respectively. The proposed BB-SPA adopts predictions of parking traffic and parking length. Compared with the traditional parking mechanism, the proposed BW-SPA, BB-SPA and PBF-SPA significantly increase the utilization of parking space and hence improves the benefit of parking lots while guaranteeing the quality of parking services. Simulation results also verify the performance improvement in terms of accumulated parking rate and service quality.

\section{References}

1. C. Roman, R. Liao, P. Ball, S. Ou and M. de Heaver, "Detecting On-Street Parking Spaces in Smart Cities: Performance Evaluation of Fixed and Mobile Sensing Systems," IEEE Transactions on Intelligent Transportation Systems, vol. 19, no. 7, pp. 2234-2245, July 2018.

2. Jong-Ho Shin, Namhun Kim, Hong-bae Jun, and Duck Young Kim, "A Dynamic Information-Based Parking Guidance for Megacities considering Both Public and Private Parking," Journal of Advanced 
Transportation, vol. 2017, pp. 1-19, July 2017.

3. Aamir Shahzad, Jae-young Choi, Naixue Xiong, Young-Gab Kim, and Malrey Lee, "Centralized Connectivity for Multiwireless Edge Computing and Cellular Platform: A Smart Vehicle Parking System," Wireless Communications and Mobile Computing, vol. 2018, pp. 1-23, Feb. 2018.

4. Surafel Luleseged Tilahun and Giovanna Di Marzo Serugendo, "Cooperative Multiagent System for Parking Availability Prediction Based on Time Varying Dynamic Markov Chains," Journal of Advanced Transportation, vol. 2017, pp. 1-14, Sept. 2017.

5. K. Banti, M. Louta and G. Karetsos, "ParkCar: A smart roadside parking application exploiting the mobile crowdsensing paradigm," 2017 8th International Conference on Information, Intelligence, Systems \& Applications (IISA), Larnaca, 2017, pp. 1-6.

6. J. Fang, A. Ma, H. Fan, M. Cai and S. Song, "Research on smart parking guidance and parking recommendation algorithm," 2017 8th IEEE International Conference on Software Engineering and Service Science (ICSESS), Beijing, 2017, pp. 209-212. 